\section{Predictive Value of Screening for Addressable Social Risk Factors}

\section{Art Jones ${ }^{1}$, Christy Harris Lemak ${ }^{2}$, Cheryl Lulias ${ }^{3}$, Todd Burk- ard $^{3}$, Beth Mc Dowell ${ }^{3}$ and Kylie Severson ${ }^{3}$}

${ }^{1}$ Health Management Associates, Chicago, USA

${ }^{2}$ Department of Health Services Administration, University of Alabama at Birmingham, USA

${ }^{3}$ Medical Home Network, Chicago, USA

\begin{abstract}
\section{Objectives}

To evaluate whether a screening assessment tool that identifies medical and social risk factors is predictive of healthcare costs in the absence of claims data. To evaluate the effectiveness of screening for social factors to target high cost beneficiaries.

\section{Study design}

A retrospective study evaluating medical and pharmacy claims costs a year after the behavioral and social risk assessment was performed.

Methods

We analyzed medical and pharmacy claims, medical and social risk assessment information, and patient enrollment data for 7,762 newly-eligible Medicaid adults. We controlled for age, gender, and common chronic diseases. We performed multivariable regressions across cost and utilization outcome measures, created risk stratification groups and assessed hospital utilization and spending based on the number and type of social factors.

\section{Results}

Although many managed care networks do not systematically collect social and behavioral risk factors in a medical setting, social risk factors are associated with an increase in healthcare costs. Healthcare costs were $\$ 65$ Per Member Per Month higher for beneficiaries who had trouble securing food, clothing, or housing (95\% Confidence Interval $[\mathrm{Cl}], \$ 10$ to $\$ 119 ; \mathrm{P}=0.02)$. The presence of $1-3$ addressable risk factors is associated with $\$ 132$ higher Per Member Per Month spending, even when both groups reported infrequent hospitalizations (95\% Confidence Interval [Cl], \$77 to $\$ 188$;
\end{abstract}

*Corresponding author: Christy Harris Lemak, Department of Health Services Administration, University of Alabama at Birmingham, USA, Tel: +1 2059757998; E-mail: lemak@uab.edu

Citation: Jones A, Lemak CH, Lulias C, Burkard T, Mc Dowell B, et al. (2017) Predictive Value of Screening for Addressable Social Risk Factors. J Community Med Public Health Care 4: 030.

Received: September 02, 2017; Accepted: October 09, 2017; Published: October 23, 2017

\begin{abstract}
$\mathrm{P}<0.001)$.
Conclusion

Social risk factors lead to an increase in healthcare cost and utilization even after controlling for past hospital utilization, chronic conditions, age, and gender. Managed care networks should routinely screen for social factors to target at-risk patients to better manage long-term healthcare costs.
\end{abstract}

Keywords: Care management solutions; Medicaid expansion; Social determinants of health

\section{Introduction}

The Medicaid expansion of the patient protection and Affordable Care Act of 2010 (ACA) enabled previously uninsured adults to buy health insurance and access a range of health care services previously unavailable to them. Though heterogeneous, the population includes a subset of patients with high medical, behavioral health, and social needs. Part of the rationale for Medicaid expansion was that identification and intervention through care management would not only improve individual health outcomes, but also reduce overall health care costs. [1]. As the Medicaid expansion population evolves, providers working with Medicaid patients may require information and data models to help anticipate costs and guide them to the most effective allocations of care management resources [2]. This paper describes the development and value of a medical and social risk assessment tool as well as the utilization and evaluation of the risk assessment tool among adults newly insured under the ACA.

Studies show that health outcomes are influenced by factors in five domains-social circumstances, behavioral patterns, environmental exposures, and genetics and health care. Health care only accounts for an estimated ten percent of the combined impact [3]. From a social determinants of health perspective, many unmet social needs can create barriers to treatment compliance and are in fact, potentially addressable either directly by health care providers; through referral to community based organization; or with improvements in community infrastructure [4-6]. Systematic risk assessment screening can detect these addressable risk factors and, when combined with historical utilization and cost data, may suggest a superior algorithm to stratify individuals for care management interventions [7]. Accordingly, CMS recently released the accountable health communities model request for proposal to test whether enhanced clinical-community linkages can improve health outcomes and reduce costs. [8].

The Medical Home Network (MHN) is an organization that manages a Medicaid ACO (MHN ACO) comprised of nine Federally Qualified Health Centers (FQHCs) and three hospital systems serving approximately 80,000 Chicago area Medicaid beneficiaries [9]. Hypothesizing that detecting and addressing barriers to treatment compliance could improve care management effectiveness, MHN designed a five-minute risk assessment tool that questions new beneficiaries about health and social risk factors and historic Emergency Department (ED) and inpatient hospital utilization. By assessing health risk using social determinants of health framework, MHN has implemented one of the largest applications of a social risk assessment among a population of newly eligible ACA Medicaid beneficiaries. 
Beginning in July 2014, MHN care management staff began systematically screening new Medicaid expansion beneficiaries. They developed a new risk stratification methodology based on the assumption that social risk factors would subsequently result in an increase of ED or inpatient services and increase future healthcare costs. MHN created a health screening tool that identifies areas of high risk Medicaid enrollees in order to assess a patient's need for care management. Risk stratification typically depends on historical claims data, which is generally unavailable for the previously uninsured and does not reflect external barriers to treatment compliance, such as addressable social determinants of health. As the Medicaid program evolves in the current political and regulatory environment, decision-makers may require additional tools and data models to identify high and rising risk beneficiaries shortly after enrollment. This can guide the most effective allocation care management resources.

The Health Risk Assessment (HRA) is a 20-question screening health risk assessment tool that can be administered in five minutes by a non-clinically licensed individual. The tool was developed based upon previous research demonstrating how health outcomes are influenced by factors beyond genetics and healthcare. The tool measures twelve addressable risk factors, including questions about access to medical care and transportation; general health and health care history; mental health and substance abuse history; and social support and needs. When scored, the HRA stratifies patients into four groups based on social factors and those historically high hospital utilizers as well as those who have minimally interacted with the health care system. This study evaluates the effectiveness of the HRA with the Medicaid expansion population by examining the financial costs of social risk factors.

\section{Methods}

To explore how medical and social risk factors impact use and spending, we used MHN ACO medical and pharmacy claims, medical and social risk assessment information, and patient enrollment data. The total potential population included 31,422 adults aged 18-67 who were newly eligible for Medicaid through the Affordable Care Act (ACA) between September 2014 and March 2015. Of these, 13,252 (42\%) completed a risk assessment and of these, 7,762 (59\%) were continuously enrolled twelve months after completing the assessment. MHN's risk assessment completion rate increased to $77 \%$ in the next six months. This group constitutes our final study sample. This population's demographic characteristics are similar to other urban newly eligible ACA Medicaid adult populations. Full descriptive data are available in the supplemental appendix.

The risk assessment tool was developed by a team of MHN clinicians and based on evidence regarding the most common chronic illnesses with potential for care management impact [10] and observed barriers to treatment compliance among MHN providers who have worked with this population for decades. It is similar to other tools [11] but focuses specifically on risk factors that care managers could mitigate by working with the patient over time.

For spending, we analyzed medical, pharmacy and total spending for the 12 months following the time when the risk assessment tool was administered. We developed a Per Member Per Month (hereinafter known as "PMPM") metric using the ratio of claims costs divided by the number of individuals in a particular group on a monthly basis. For utilization, we analyzed visit counts for the Emergency Room department, admission counts, readmission counts, and length of stay for inpatient hospital in the 12 months following the time when the risk assessment tool was administered. We controlled for gender and whether or not they reported having diabetes or asthma, the most commonly reported chronic conditions within our population (diabetes at $15 \%$; asthma at $9 \%$ ).

We also performed multivariable regressions across all the different outcome measures, created risk stratification groups and assessed relative use and spending by category. Complete descriptive statistics and regression results are available in the supplemental appendix.

We recognize the following limitations to our analyses. First, there is no control group for the study. In addition, results are specific to the Chicago (Cook County) Medicaid population served by MHN. We controlled for two ambulatory-sensitive diseases that were most commonly reported. None of the additional ambulatory-sensitive diseases impacted more than $5 \%$ of the population. Furthermore, all information in the risk assessment is self-reported and not confirmed by other sources.

\section{Results}

As shown in Exhibit 1 (Supplementary File 1), we found that the health and social factors measured in the risk assessment were present among many newly eligible Medicaid expansion adult beneficiaries. For example: $5 \%$ reported not feeling safe at home, $13 \%$ have trouble making appointments, $15 \%$ have trouble paying for prescriptions, $17 \%$ have limited transportation to healthcare services, and almost $24 \%$ need help getting food, clothing, and housing. BMI over 30 (a medical risk factor) is most common, occurring among $42 \%$ of the population. Despite its prevalence, it was not found to be statistically significant after controlling for other social risk factors.

Many risk factors outside of the traditional medical model may be associated with higher utilization and costs. Exhibit 2 (Supplementary File 1) highlights significant, discrete relationships between addressable factors and subsequent utilization and PMPM spending for the twelve months following the risk assessment. Some addressable factors are associated with greater medical and pharmacy PMPM spending, such as needing help getting food, clothing or housing, reporting fair or poor health status, and experiencing transportation challenges. The six most common addressable factors were all associated with higher hospital readmissions; most of them were linked significantly to greater subsequent inpatient stays and ED visits.

Beneficiaries who are homeless or living in a shelter experienced greater healthcare utilization than others. Although they only represent $1 \%$ of this population, beneficiaries who are homeless or live in a shelter had significantly more ED visits, inpatient admissions and hospital readmissions. Beneficiaries with housing needs may be further represented in the quarter of our sample who reported needing help securing food, clothing or housing; beneficiaries who needed help securing food, clothing, and housing had healthcare costs that were \$65 PMPM higher than those who do not have this addressable risk factor, all else equal. Beneficiaries with additional social factors may see an additional increase in costs. For example, beneficiaries who report fair or poor health in addition to help securing food, clothing, and housing will see a PMPM increase of \$259 (\$198 attributed to reporting poor health; the remainder attributed to the need for food, clothing, and housing).

As shown in Exhibit 3 (Supplementary File 1), the presence of any addressable risk factor is associated with significantly higher 
allowed PMPM spending despite a history of hospital utilization. For example, among those with a history of high prior hospital utilization, those with six or more addressable risk factors experience a $75 \%$ higher PMPM spending than those with no risk factors. We tested the stratification system that was based on a combination of utilization and the number of addressable risk factors (Exhibit 4, Supplementary File 1). Our goal was to go beyond the traditional identification of high risk beneficiaries through chronic disease burden by identifying, quantifying, and addressing social risk factors in addition to medical issues. This would allow care managers to intervene and prevent the "rising risk" beneficiaries from ever becoming high cost.

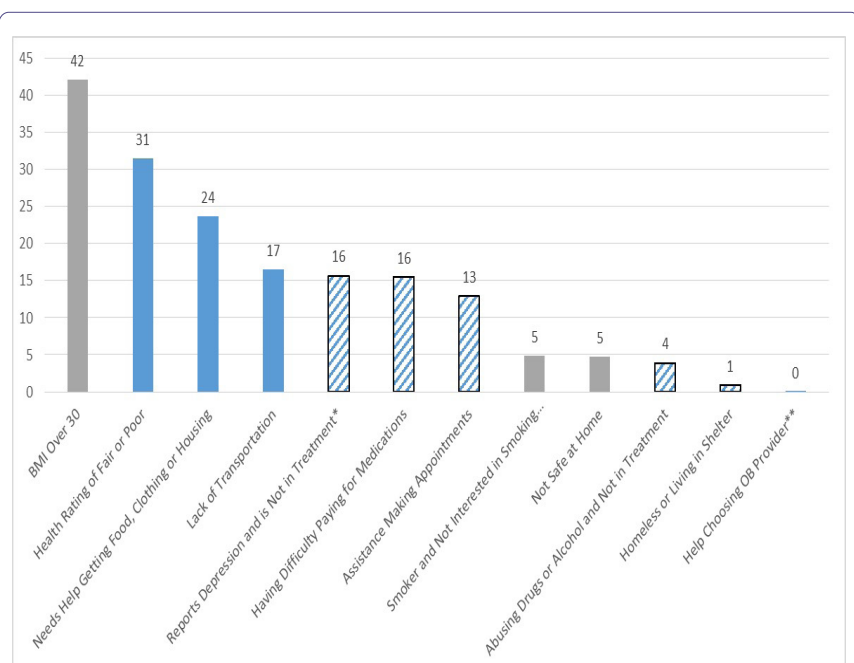

Exhibit 1: Prevalence of addressable risk factors associated with higher subsequent utilization and medical PMPM spending.

Source/Notes: $\mathrm{N}=7,762$ adult Medicaid beneficiaries with 12-months continuous enrollment after completing health risk assessment; $p<0.05$; controlling for age, gender, asthma and diabetes.

Grey shading=no significant relationship with costs and utilization; Blue shading=significantly higher medical PMPM costs and utilization; Blue stripes=significant change in utilization.

*The risk factor "reports depression and is not in treatment" showed a statistically significant decrease in utilization.

**The risk factor "help choosing OB provider" is not statistically significant.

Source: Analysis of MHN ACO Data

Notes: Regression results available in supplemental appendix.

As shown in Exhibit 4 (Supplementary File 1), setting the low risk category (no social factors and low prior utilization) as the baseline spend, we observe that for those without historically high hospital utilization, the presence of 1-3 risk factors was associated with higher future spending by $61 \%$; $4-5$ risk factors is associated with $95 \%$ higher future spending; 6 or more with $86 \%$ higher future spending. Subsequent ED utilization is correlated significantly with the number of addressable risk factors as is, for the most part, future inpatient admissions.

MHN uses results reported on the risk assessment to allocate care management resources. They deploy a multi-disciplinary, team-based approach that includes care management staff. Each primary care practice collaborates with community partners to address and attempt to resolve the individual's self-identified barriers to care. For example, Joe (not the patient's real name) is a 40-year-old male assigned to Lawndale Christian Health Center. Lawndale's care team reached out to the patient to complete his risk assessment. In addition to a history of high hospitalizations and ED use, care managers identified Joe as having multiple addressable risk factors-including trouble with transportation, help with food/housing/clothing, mental health issues, and substance abuse issues. Joe's care manager continued to work with him over the next year. Once Joe trusted his care team, he admitted that he was unwilling to allow the care manager to visit his home because he was ashamed of his living conditions (he was squatting in a vacant building). Lawndale's care team worked with Joe to secure a referral for emergency housing and he was eventually accepted into the Department for Housing and urban development housing choice voucher program and obtained stable housing. He continues to improve his care through engagement with the care team at Lawndale and has stayed out of the emergency room for the past 6 months.

\begin{tabular}{|c|c|c|c|c|c|c|c|}
\hline \multirow{2}{*}{\multicolumn{2}{|c|}{ HRA Risk Factors }} & \multicolumn{3}{|c|}{ PMPM } & \multicolumn{3}{|c|}{ Utilization } \\
\hline & & Med Only & Rx Only & Med $+R_{s}$ & $\underline{\underline{P}}$ & Readmits & $\underline{E R}$ \\
\hline \multicolumn{2}{|c|}{$\begin{array}{l}\text { Needs Help Getting Food, Clothing or } \\
\text { Housing }\end{array}$} & $\overline{\checkmark \checkmark}$ & $\bar{x}$ & $\checkmark$ & $\checkmark v$ & $\checkmark \checkmark$ & $\sqrt{ } \checkmark \checkmark \checkmark$ \\
\hline \multicolumn{2}{|c|}{ Health Rating of Fair or Poor } & $\checkmark \checkmark \checkmark$ & $\checkmark \checkmark$ & $\checkmark d \checkmark$ & $a d t$ & $\checkmark d$ & $a d \gamma$ \\
\hline \multirow{2}{*}{\multicolumn{2}{|c|}{$\begin{array}{l}\text { Lack of Transportation } \\
\text { Abusing Drugs or Alcohol and Not in } \\
\text { Treatment }\end{array}$}} & $\checkmark$ & $\mathrm{x}$ & $\mathrm{x}$ & $\checkmark d$ & $\checkmark$ & $\checkmark \checkmark \checkmark$ \\
\hline & & $x$ & $\mathrm{x}$ & $\mathrm{x}$ & $\checkmark \checkmark$ & $\checkmark \checkmark$ & $\checkmark$ \\
\hline \multicolumn{2}{|c|}{ Homeless or Living in Shelter } & $x$ & $\mathrm{x}$ & $x$ & $\Delta d \checkmark$ & $a d q$ & $a d v$ \\
\hline \multirow{2}{*}{\multicolumn{2}{|c|}{$\begin{array}{l}\text { Assistance Making Appointments } \\
\text { Having Diffliculty Paying for Medications }\end{array}$}} & $\mathrm{x}$ & $x$ & $\mathrm{x}$ & $x$ & $\mathrm{x}$ & $\checkmark$ \\
\hline & & $\mathrm{x}$ & $\mathrm{x}$ & $\mathrm{x}$ & $x$ & $\checkmark$ & $x$ \\
\hline \multicolumn{2}{|c|}{ Reports Depression and is Not in Treatment } & $\mathrm{x}$ & $\mathrm{x}$ & $x$ & $\downarrow$ & $\downarrow$ & $\mathrm{x}$ \\
\hline$x$ & \multicolumn{2}{|l|}{ Not Significant } & & & & & \\
\hline$\checkmark$ & \multicolumn{2}{|c|}{ Statistically significant increase; $p<0.05$} & & & & & \\
\hline $\begin{array}{l}-1 \\
\checkmark \checkmark \\
\checkmark \checkmark \checkmark\end{array}$ & \multicolumn{2}{|c|}{$\begin{array}{l}\text { Statistically significant increase; } p<0.01 \\
\text { Statistically significant increase; } ; \text { p } \quad 0.001\end{array}$} & & & & & \\
\hline$\downarrow$ & \multicolumn{2}{|c|}{ Statistically significant decrease; $p<0.05$} & & & & & \\
\hline
\end{tabular}

Exhibit 2: Significant risk factors by utilization and spending subsets.

Source/Notes: $\mathrm{N}=7,762$ adult Medicaid beneficiaries with 12-months continuous enrollment after completing health risk assessment; p-value less than 0.05, 0.01, 0.001; controlling for age, gender, asthma and diabetes.

*All but one risk factor significantly increased utilization. The risk factor "reports depression and is not in treatment" showed a statistically significant decrease in utilization.

Source: Analysis of MHN ACO Data

Notes: Regression results available in supplemental appendix.

Sue (not the patient's real name) is a female in her mid-30's who was assigned to Esperanza Health Center for primary care. When the care manager from Esperanza first met Sue, she was admitted as an inpatient to the hospital and was sedated and non-verbal. The care manager followed up with Sue her family to schedule a hospital follow-up appointment at Esperanza. The care manager completed a risk assessment for Sue and learned that lack of transportation was a barrier for completing medical appointments. The care manager provided the patient with information about how to schedule free transportation to medical appointments. With regular access to her medications, Sue's functional status improved. She was able to receive follow-up care in a lower acuity clinic setting and has stayed out of the hospital.

These are two examples of the type of care coordination that is needed to address the complex risk factors at play for the rising risk population of newly eligible Medicaid beneficiaries. The care team can better direct resources to help Joe and Sue with knowledge of addressable social risk factors. MHN providers are collaborating to maintain active engagement with patients and most effectively assign resources that go beyond medical care and extends to transportation, food, and housing. The work includes licensed and unlicensed professionals as well as a network of community partners outside the healthcare system. 


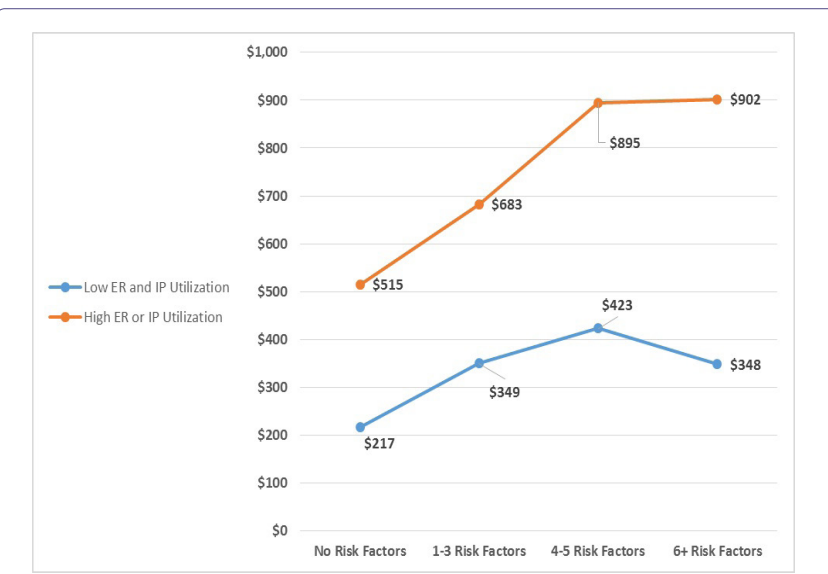

Exhibit 3: Total allowed PMPM Spending by number of addressable risk factors: comparing those with and without a history of high Emergency Room or inpatient utilization.

Source/Notes: Analysis of MHN ACO Data; Regression results available in supplemental appendix.

$\mathrm{N}=7,762$ adult Medicaid beneficiaries with 12-months continuous enrollment after completing health risk assessment; $\mathrm{p}<0.05$; controlling for age, gender, asthma and diabetes.

High ED utilization is defined as beneficiaries who self-reported having 3 or more ED visits in the last 6 months, while low ED utilization is defined as beneficiaries who denied having ER utilization at this level. High IP utilization is defined as beneficiaries who self-reported either (a) having 1 or more hospitalizations for ambulatory-sensitive chronic conditions (e.g., heart failure, asthma, diabetes) or (b) self-reported having 2 or more hospitalizations in past 12 months for any other condition. Low IP utilization is defined as beneficiaries who denied having IP utilization at this level.

\section{Conclusion}

This analysis reveals statistically significant relationships between addressable risk factors and subsequent hospital utilization and costs among newly eligible Medicaid expansion beneficiaries. Future studies will investigate which specific care management approaches are most effective in engaging rising risk patients, driving care to the most appropriate locations, and reducing the total cost of care. We are also exploring how our measures of addressable risk and targeted case management approaches allow us to achieve better health outcomes in all patient populations. We will also investigate how care management interventions may impact social factors and related risk levels overtime.

Especially in light of the ongoing discussion around the fate of Medicaid expansion, this study importantly demonstrates that limited care management resources can be directed at those most likely to benefit [12]. It supports the assumption that certain barriers to compliance with treatment plans are important determinants of future total cost of care and can be addressed at the individual patient level by the care manager either directly or by referral to social service agencies. When aggregated, the prevalence of these risk factors can inform public health efforts to address them systemically as well. Future analyses on this population may stratify the population by age and gender, rather than controlling for these variables as developed in the current analysis.

\begin{tabular}{|c|c|c|c|c|c|c|c|c|}
\hline & \multicolumn{4}{|c|}{ Self -reported on Risk Assessment } & \multicolumn{3}{c|}{ Based on claims Data 12 Months After Risk Assessment } \\
\hline Risk Levels & $\begin{array}{c}\text { Number of Risk } \\
\text { Factor }\end{array}$ & Utilization & $\begin{array}{c}\text { Count of } \\
\text { Beneficiaries }\end{array}$ & $\%$ of Total & $\begin{array}{c}\text { ER } \\
\text { Visits/1000 }\end{array}$ & $\begin{array}{c}\text { Inpatient } \\
\text { Admits/1000 }\end{array}$ & Total PMPM & Relative Cost \\
\hline Low & & Low & 1,606 & $20.70 \%$ & 415 & 57 & $\$ 127$ & $100 \%$ \\
\hline Low by social factors & $1-3$ & Low & 4,181 & $53.90 \%$ & 620 & 96 & $\$ 349$ & $161 \%$ \\
\hline Medium by social factors & $4-5$ & Low & 663 & $8.50 \%$ & 742 & 143 & $\$ 423$ & $195 \%$ \\
\hline Medium by utilization & $0-5$ & High ED; Low IP & 320 & $4.10 \%$ & 1856 & 281 & $\$ 480$ & $221 \%$ \\
\hline High by social factors & $6+$ & Low & 127 & $1.60 \%$ & 835 & 126 & $\$ 405$ & $186 \%$ \\
\hline High by utilization & Any & High IP & 865 & $11.10 \%$ & 1653 & 680 & $\$ 821$ & $378 \%$ \\
\hline Total & & & 7,762 & $100.00 \%$ & 758 & 165 & $\$ 387$ & \\
\hline
\end{tabular}

Exhibit 4: Using MHN's risk stratification model: utilization and PMPM spending by risk category.

Source/Notes: Analysis of MHN ACO Data; Regression results available in supplemental appendix.

$\mathrm{N}=7,762$ adult Medicaid beneficiaries with 12-months continuous enrollment after completing health risk assessment; $\mathrm{p}<.05$; controlling for age, gender, asthma and diabetes.

Percentages in the column "relative cost" are anchored to the "low" risk level.

High ER utilization is defined as beneficiaries who self-reported having 3 or more ED visits in the last 6 months, while low ED utilization is defined as beneficiaries who denied having ED utilization at this level.

High IP utilization is defined as beneficiaries who self-reported either (a) having 1 or more hospitalizations for ambulatory-sensitive chronic conditions (e.g., heart failure, asthma, diabetes) or (b) self-reported having 2 or more hospitalizations in past 12 months for any other condition. Low IP utilization is defined as beneficiaries who denied having IP utilization at this level.

Source/Notes: N=7,762 adult Medicaid beneficiaries with 12-months continuous enrollment after completing health risk assessment; $p<0.05$; controlling for age, gender, asthma and diabetes. High emergency room use defined as beneficiaries who had had more than 3 ER visits in the last 6 months. High inpatient utilization is defined as beneficiaries who reported either (1) having 1 or more hospitalizations for Heart Failure, Heart Attack, Asthma, COPD, Emphysema, Diabetes, Chronic Mental Health, and/or Substance Abuse OR (2) reported having 2 or more hospitalizations in past 12 months for any other conditions.

Source: Analysis of MHN ACO data

Notes: Regression results available in supplemental appendix. 


\section{Public Health Implications}

We have demonstrated that there are addressable social risk factors that can predict the likelihood of future hospital utilization and global costs among adult Medicaid expansion beneficiaries. Our research augments the traditional claims-based risk stratification algorithm to guide the deployment of care management resources, especially for those working in the safety net. This new evidence-based tool can be used to help create patient identified goals for individualized care plans and enhance the likelihood of improved outcomes, including a positive return on the investment in care management. We believe these findings represent an important step toward developing a more fully integrated, community-based model of care that is accountable to patients' holistic well-being by addressing medical, behavioral, and social needs. Screening for and addressing barriers to treatment plan compliance is central to identifying high risk Medicaid expansion beneficiaries and provide a path to improving population health.

\section{References}

1. Peikes D, Chen A, Schore J, Brown R (2009) Effects of care coordination on hospitalization, quality of care, and health care expenditures among Medicare beneficiaries: 15 randomized trials. JAMA 301: 603-618.

2. Haas LR, Takahashi PY, Shah ND, Stroebel RJ, Bernard ME, et al. (2013) Risk-stratification methods for identifying patients for care coordination. Am J Manag Care 19: 725-735.

3. Schroeder SA (2007) We can do better-improving the health of the American people. The New England Journal of Medicine 357: 1221-1228.

4. Thompson B, Molina Y, Viswanath K, Warnecke R, Prelip ML (2016) Strategies to empower communities to reduce health disparities. Health Aff (Millwood) 35: 1424-1428.
5. Purnell TS, Calhoun EA, Golden SH, Halladay JR, Krok-Schoen JL, et al. (2016) Achieving health equity: closing the gaps in health care disparities, interventions, and research. Health Aff (Millwood) 35: 1410-1415.

6. The Menges Group (2014) Positively Iimpacting social determinants of health: how safety net health plans lead the way. Association for Community Affiliated Plans, Washington DC, USA.

7. Pratt R, Hibberd C, Cameron IM, Maxwell M (2015) The Patient Centered Assessment Method (PCAM): integrating the social dimensions of health into primary care. Journal of Comorbidity 5: 110-119.

8. Alley DE, Asomugha CN, Conway PH, Sanghavi DM (2016) Accountable health communities-addressing social needs through Medicare and Medicaid. The New England Journal of Medicine 374: 8-11.

9. Medical Home Network (2016) Building better partnerships for better health. Medical Home Network, Illinois, USA.

10. Health Care Transformation Task Force (2015) Proactively Identifying the High Cost Population. Health Care Transformation Task Force, Carolina, USA.

11. Kresge Foundation (2015) Health Centers Addressing the Social Determinants of Health. Kresge Foundation, California, USA.

12. Sommers BD, Blendon RJ, Orav J, Epstein AM (2016) Changes in utilization and health among low-income adults after medicaid expansion or expanded private insurance. JAMA Intern Med 176: 1501-1509.

For supplementary file 1 please click below link

http://www.heraldopenaccess.us/fulltext/Community-Medicine-\&-Public-Health-Care/Supplementary_File_Regressions_Supporting_Exhibits.php 